\title{
TIPOS DO HERBÁRIO DO JARDIM BOTÂNICO DO RIO DE JANEIRO MELASTOMATACEAE - V
}

\author{
L. D'A. FREIRE DE CARVALHO $\left({ }^{*}\right)$ \\ SÔNIA L. PEIXOTO (**) \\ Seção de Botânica Sistemática \\ Jardim Botânico, Rio de Janeiro
}

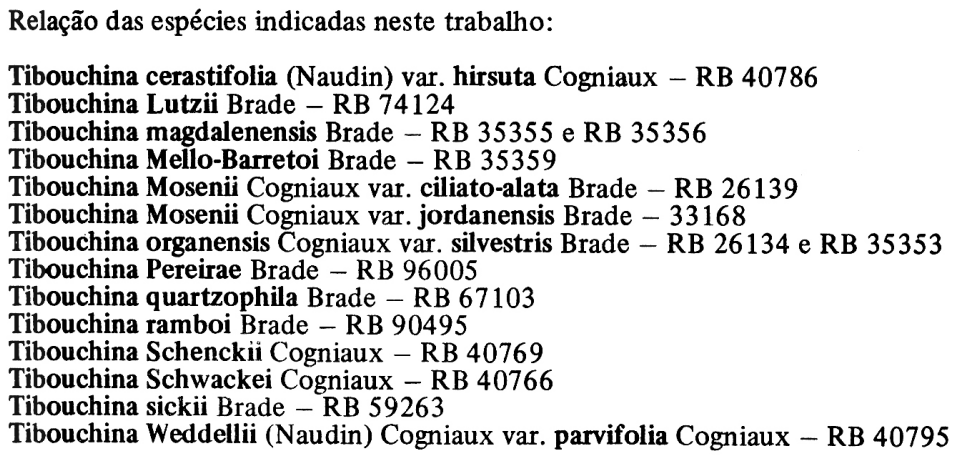

61 - Tibouchina cerastifolia (Naudin) Cogniaux var. hirsuta Cogniaux in De Candolle Monogr. Phanerog. Prod. 7: 1177.1891. "In prov. S. Catharina ad Serra do Mar Prope Sao-Bento (Anna Schwacke)".

$$
\text { EXEMPLAR -RB } 40786 \ldots \ldots \ldots \ldots \text { ISOTYPUS (FOTO 1) }
$$

Sched.: F1. pallide rosei (11.II.1890). Herb. Schwacke 6916.

Obs.: Posteriormente doado para o Herbario Damazio.

62 - Tibouchina Lutzii Brade, Mem. Inst. Oswaldo Cruz 53 (2-3 e 4): 353, est. 1, figs. 1-6. 1955. "Habitat: Brasilia, Estado de São Paulo, Serra da Bocaina, leg. Adolpho et Bertha Lutz N.o 1962, Dez. de 1930. Herbário Prof. Adolpho Lutz N. ${ }^{\circ} 1.962$ - Serra da Bocaina, $1.700 \mathrm{msn}$ do mar leg. A. C. Brade N.O 20.675 - 21.4.1951 ("Typus"), Herbário Jardim Botânico do Rio de Janeiro N.0 $74.124 "$ ".

$$
\text { EXEMPLAR }-\mathrm{RB} 74124 \ldots \ldots \ldots \text { HOLOTYPUS *** (FOTO 2) }
$$

Sched.: arbusto 1-1,5 m, bordo da mata, fl. roxa.

63 - Tibouchina magdalenensis Brade, Arch. Inst. Biol. Veget. 4 (1): 74, est. 5. 1938. "Habitat: Brasilia. Estado do Rio de Janeiro, Sta. Magdalena, Pedra Dubois $1200 \mathrm{msn}$ do mar. Leg. Santos Lima \& Brade 13.237, 28-II-1934. Typus Herbario Jardim Botânico Rio de Janeiro N. 35.355. Sta. Magdalena, Morro da Estação $800 \mathrm{msn}$ do mar nos rochedos. Leg. Santos Lima \& Brade 14.264. II-1935. Herbario Jardim Botânico Rio de Janeiro N. 35.356".

(*) Pesquisador do Jardim Botânico (R J) e do Conselho de Desenvolvimento Científico e Tecnológico.

(“") Estagiária do Jardim Botânico (RJ).

(“*) Determinada por J. J. Wurdack.

Rodriguésia

Rio de Janeiro
Vol. XXXII - n. ${ }^{0} 55$

1980 
EXEMPLAR - RB $35355 \ldots \ldots \ldots$ HOLOTYPUS ***(FOTO 3)

Sched.: Subarbusto, fl. roxas.

EXEMPLAR - RB $35356 \ldots \ldots \ldots$ PARATYPUS (FOTO 4)

Sched.: arbusto nos rochedos, fl. roxas.

64 - Tibouchina Mello-Barretoi Brade, Arch. Inst. Biol. Veget. 4 (1): 76, est. 7. 1938. "Habitat: Brasilia. Minas Geraes, Serra do Cipó. Leg. Mello Barreto N. 1.168 \& Brade N. 14.752. 15-IV-1935. Typus Herbario Jardim Botânico Rio de Janeiro N. 35.359".

EXEMPLAR - RB $35359 \ldots \ldots \ldots$. . . . . . HOLOTYPUS ***FOTO 5)

Sched.: fl. violácea.

65 - Tibouchina Mosenii Cogniaux var. ciliato-alata Brade, Arch. Inst. Biol. Veget. 4 (1): 77.1938. "Habitat: Brasilia. Estado do Rio de Janeiro Itatiaya Maromba $1.000 \mathrm{msn}$ do mar. Leg. A. C. Brade 14.615. - 22.V.1935. Herbario do Jardim Botânico Rio de Janeiro N. 26.139".

EXEMPLAR - RB $26139 \ldots \ldots \ldots$. . . . . . HOLOTYPUS (FOTOS 6 e 7)

Sched.: arbusto sobre rochedos, fl. roxa.

66 - Tibouchina Mosenii Cogniaux var. jordanensis Brade, Arch. Inst. Biol. Veget. 4 (1): 77.1938. "Habitat: Brasilia; Estado de São Paulo, Campos de Jordão. Leg. L. Danstyák IV.1937. Herb. Jard. Bot. 33.168".

$$
\text { EXEMPLAR - RB } 33168 \ldots \ldots \ldots \text { HOLOTYPUS (FOTO 8) }
$$

67 - Tibouchina organensis Cogniaux var. silvestris Brade, Arch. Inst. Biol. Veget. 4 (1): 72. 1938. "Habitat: Brasilia. Estado do Rio de Janeiro, Itatiaya km 12. $1700 \mathrm{~m}$. - Leg. P. Campos Porto n. 2.235. 14-IV-1932. J. B. 35.353; idem Brade n. 12.682. Aug. 1933. - Typus Herbario Jardim Botânico Rio de Janeiro n. 26.134".

EXEMPLAR - RB 26134 . . . . . . . . HOLOTYPUS (FOTO 9)

Sched.: peqn. árvore.

EXEMPLAR - RB $35353 \ldots \ldots \ldots$ PARATYPUS (FOTO 10)

68 - Tibouchina Pereirae Brade \& Markgraf, Arq. Jard. Bot. 17: 46, est. 2, figs. 1-10.1959/1961. "Habitat: Brasília - Estado da Bahia, entre Lençóes e Itaberaba; leg. EDMUNDO PEREIRA n. 2.062; 15-9-1956. "Typus": Herbarium Bradeanum n. 3.467. "Isotypus" Jardim Botânico do Rio de Janeiro n. 96.005".

$$
\text { EXEMPLAR - RB } 96005 \ldots \ldots \ldots \text { ISOTYPUS (FOTO 11) }
$$

Sched.: arb. de fl. violacea

69 - Tibouchina quartzophila Brade, Arq. Jard. Bot. 14: 219, est. 6, figs. 1-7. 1956. "Habitat: Brasília. Estado do Espírito Santo: Vargem Alta, Município Cachoeiro do Itapemirim, Morro do Sal $600 \mathrm{msn}$ do mar. Leg. A. C. Brade N.0 19767 - 6.V.1949. Typus Herbário Jardim Botânico do Rio de Janeiro N. 67103".

$$
\text { EXEMPLAR - RB } 67103 \ldots \ldots \ldots \text { HOLOTYPUS }{ }^{* * *}(\text { FOTOS } 12 e
$$

Sched.: arbusto $1-1,5 \mathrm{~m}, \mathrm{fl}$. alvas.

70 - Tibouchina ramboi Brade, Sellowia 8: 367, est. 1., figs. 1-6. 1957. "Habitat: Brasília. Estado do Rio Grande do Sul, Serra da Rocinha, pr., Bom Jesus, "in silvula nebulari". Leg. Balduino Rambo S. J. 3.2.1953. N. 0 53.871, "Typus" in Herbario Anchieta, Colégio Anchieta, Porto Alegre. "Coty-" pus" Herbário Jardim Botânico do Rio de Janeiro N.0 90495 - Fragmento in Herbario A. C. Brade". 
EXEMPLAR - RB 90495

Sched.: Frutex 2-metralis.

71 - Tíbouchina Schenckii Cogniaux, Bull. Torrey bot. C1. 17: 54.1890 et in De Candolle e Monogr. Phanerog. Prod. 7: 207. 1891. "In Brasilia ad Serra do Picu inter prov. Rio de Janeiro, S. Paulo et Minas Geraes (Schenck)".

EXEMPLAR - RB $40769 \ldots \ldots \ldots$. . . . . . ISOTYPUS (FOTO 15)

Sched.: Blüt. violett. Sehr hfgr Strauch in den hoheren Regionen der Serra do Picú, leg. H. Schenck, 11.12.1886. Herb. brasil 1487 .

Obs.: Ex Herb. Damazio.

72 - Tibouchina Schwackei Cogniaux, Bull. Torrey bot. Cl. 17: 54.1890 et in De Candolle Monogr. Phanerog. Prod. 7: 206. 1891. "In Brasiliae prov. Rio de Janeiro (Glaziou n. 16802)".

EXEMPLAR - RB $40766 \ldots \ldots \ldots$. . . . . . ISOTYPUS (FOTO 16)

Sched.: Alto Macahé de Nova Friburgo.

Obs.: Ex Herb. Damazio.

73 - Tibouchina sickii Brade, Arq. Jard. Bot. 14: 217, est. 4, figs. 1-7. 1956. "Habitat: Brasília. Estado de Mato Grosso: Xavantina, Rio das Mortes. Leg. Dr. Helmut Sick da Fundação Brasil Central, N. B. 283. Fev. de 1947. Typus: Herbário Jardim Botânico do Rio de Janeiro N.o 59263".

EXEMPLAR - RB $59263 \ldots \ldots \ldots$. . . . . . . HOLOTYPUS (FOTO 17)

74 - Tibouchina Weddellii (Naudin) Cogniaux var. parvifolia Cogniaux in De Candolle Monogr. Phanerog. Prod. 7: 1175. 1891. "In prov. Rio de Janeiro ad Serra dos Orgaos (de Moura)".

EXEMPLAR - RB $40795 \ldots \ldots \ldots \ldots$. . . . . ISOTYPUS (FOTO 18)

Sched.: leg. J. T. de Moura (II.1890). Herb. Schwacke.

Obs.: Ex Herb. Damazio.

NOTA: As fotografias foram tiradas pelas autoras e as cópias pelo Sr. Mário Silva, fotógrafo do Jardim Botânico do Rio de Janeiro. 


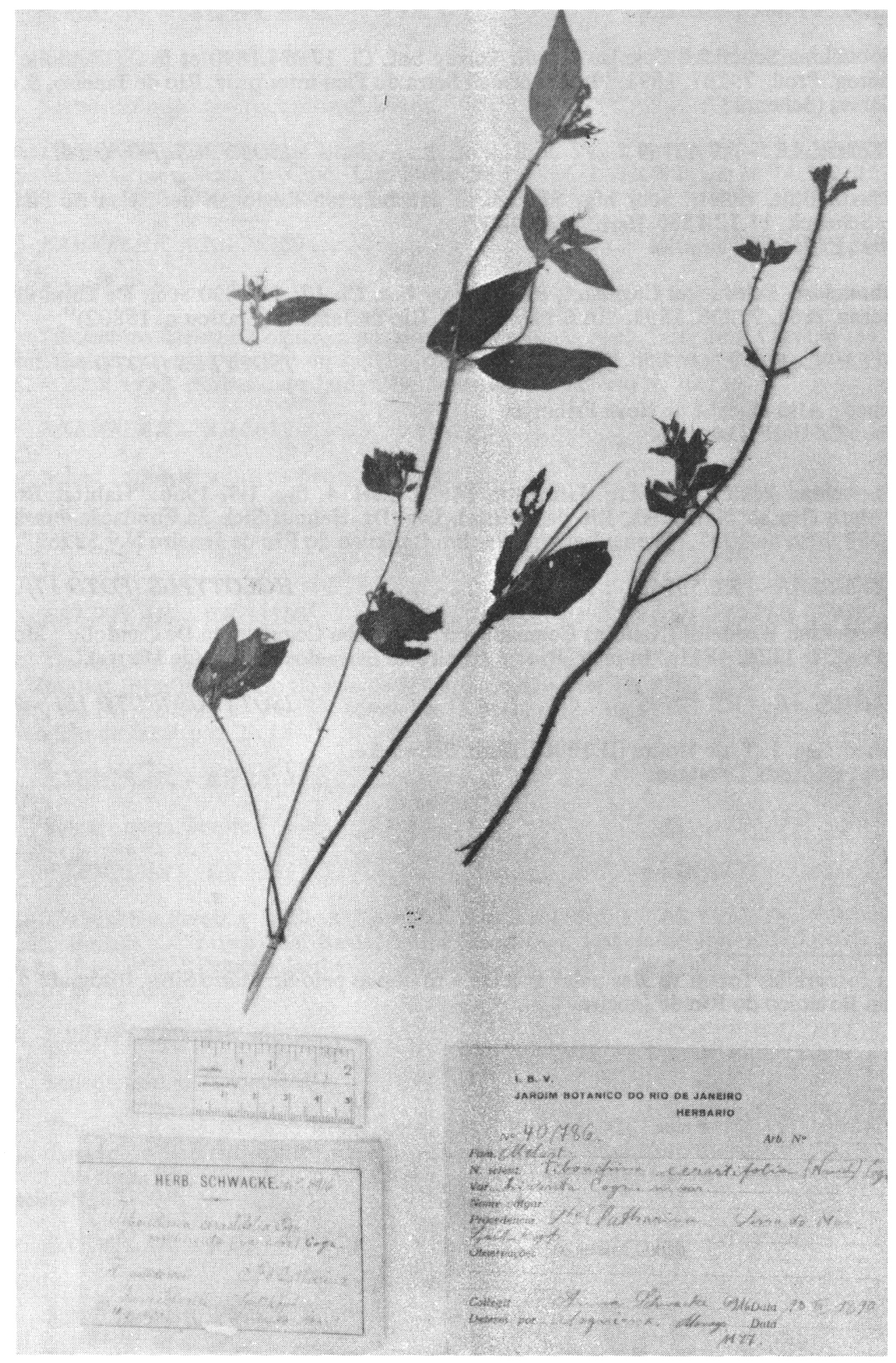

Foto 1 - Tibouchina cerastifolia (Naudin) var. hirsuta Cogniaux 


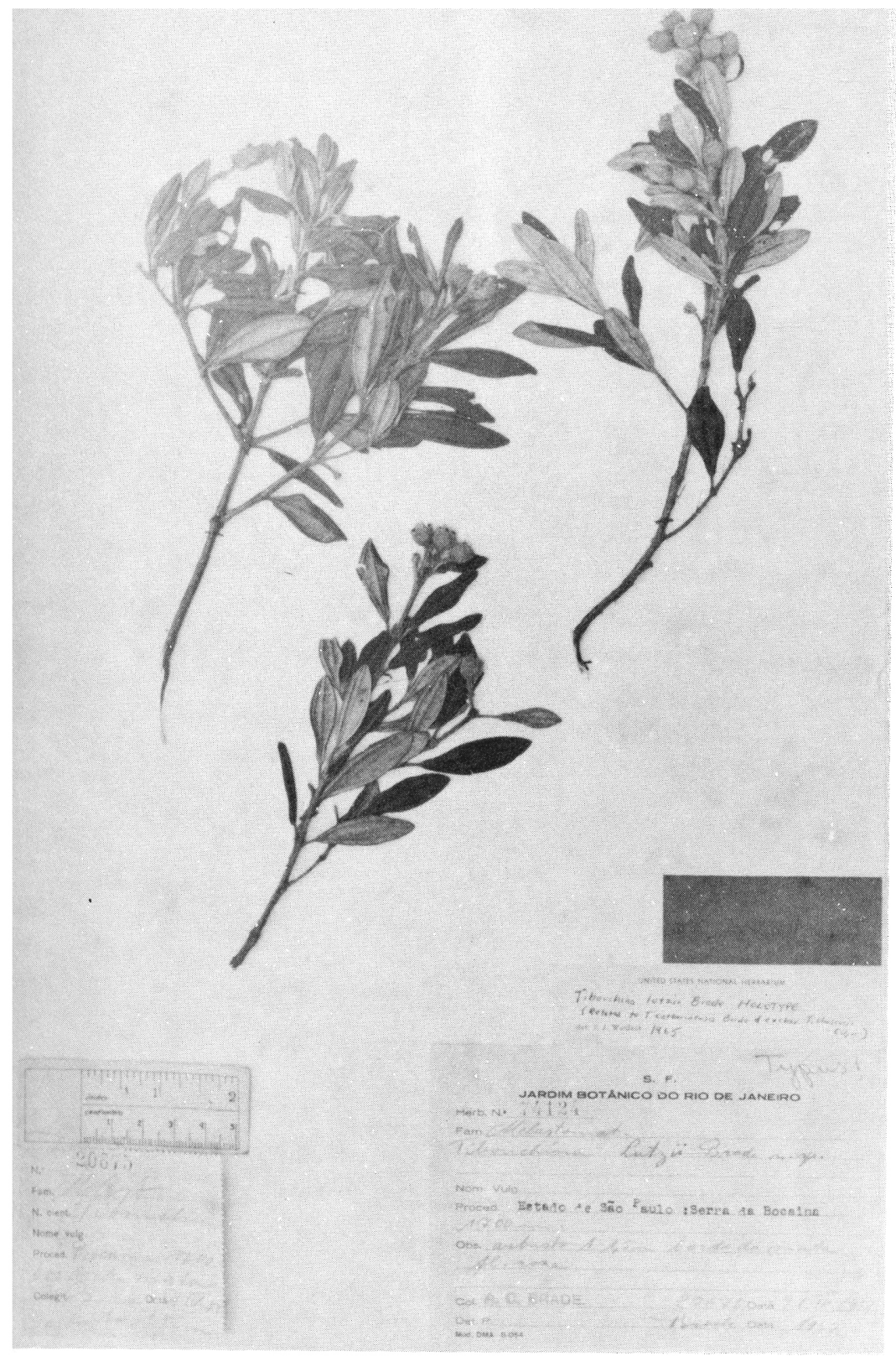

Foto 2 - Tibouchina lutzii Brade 


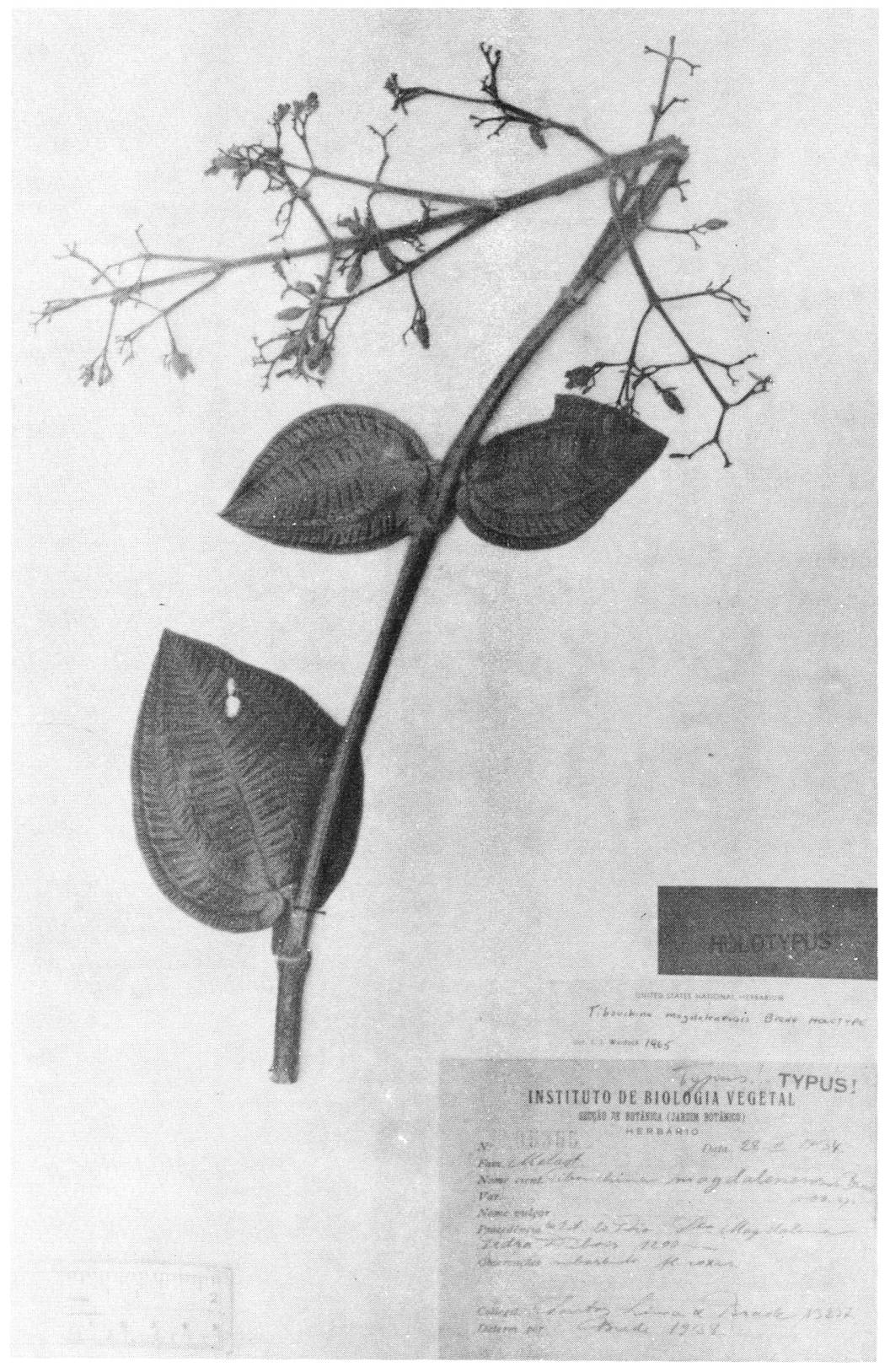

Foto 3 - Tibouchina magdalenensis Brade 


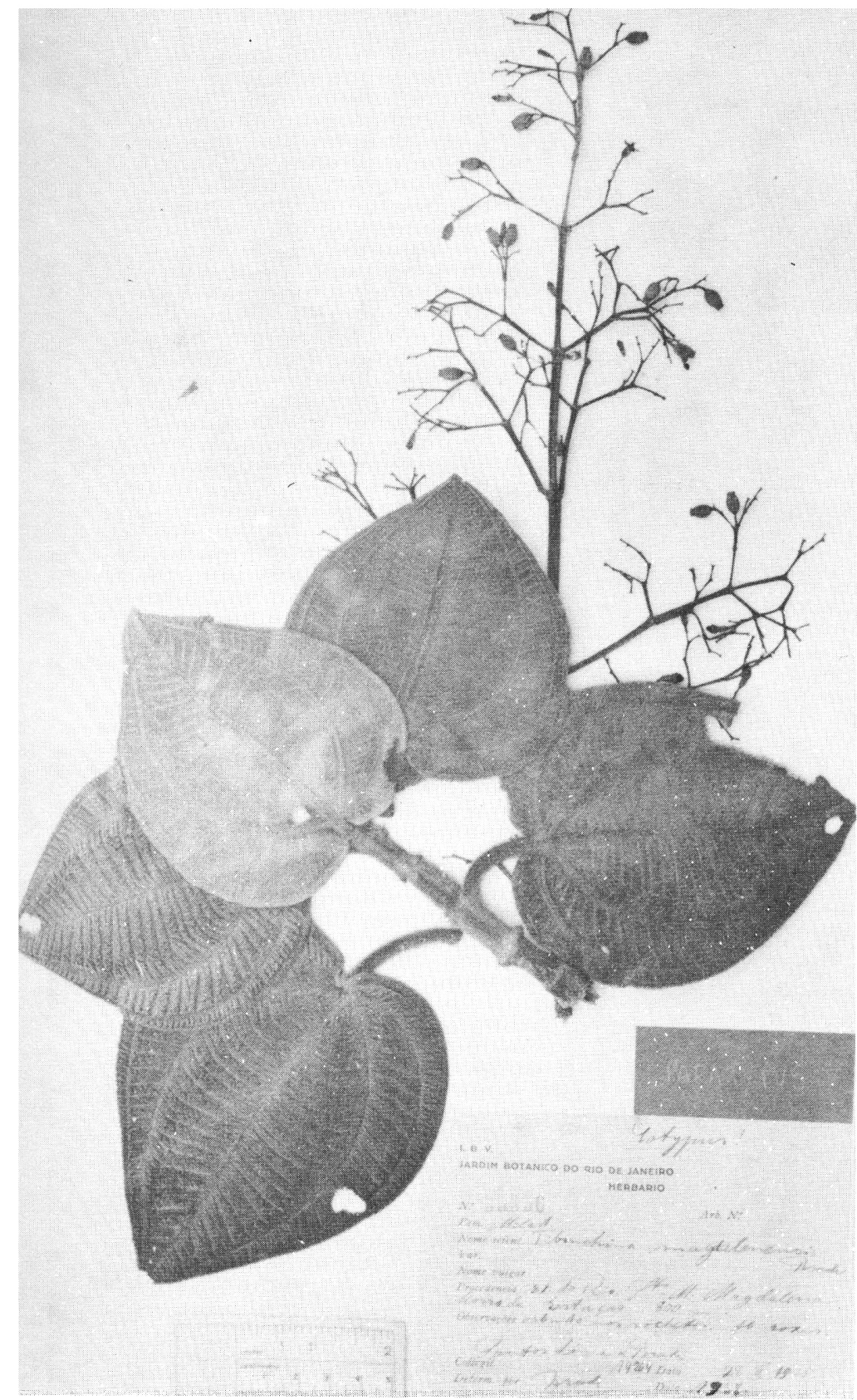

Foto 4 - Tibouchina magdalenensis Brade 


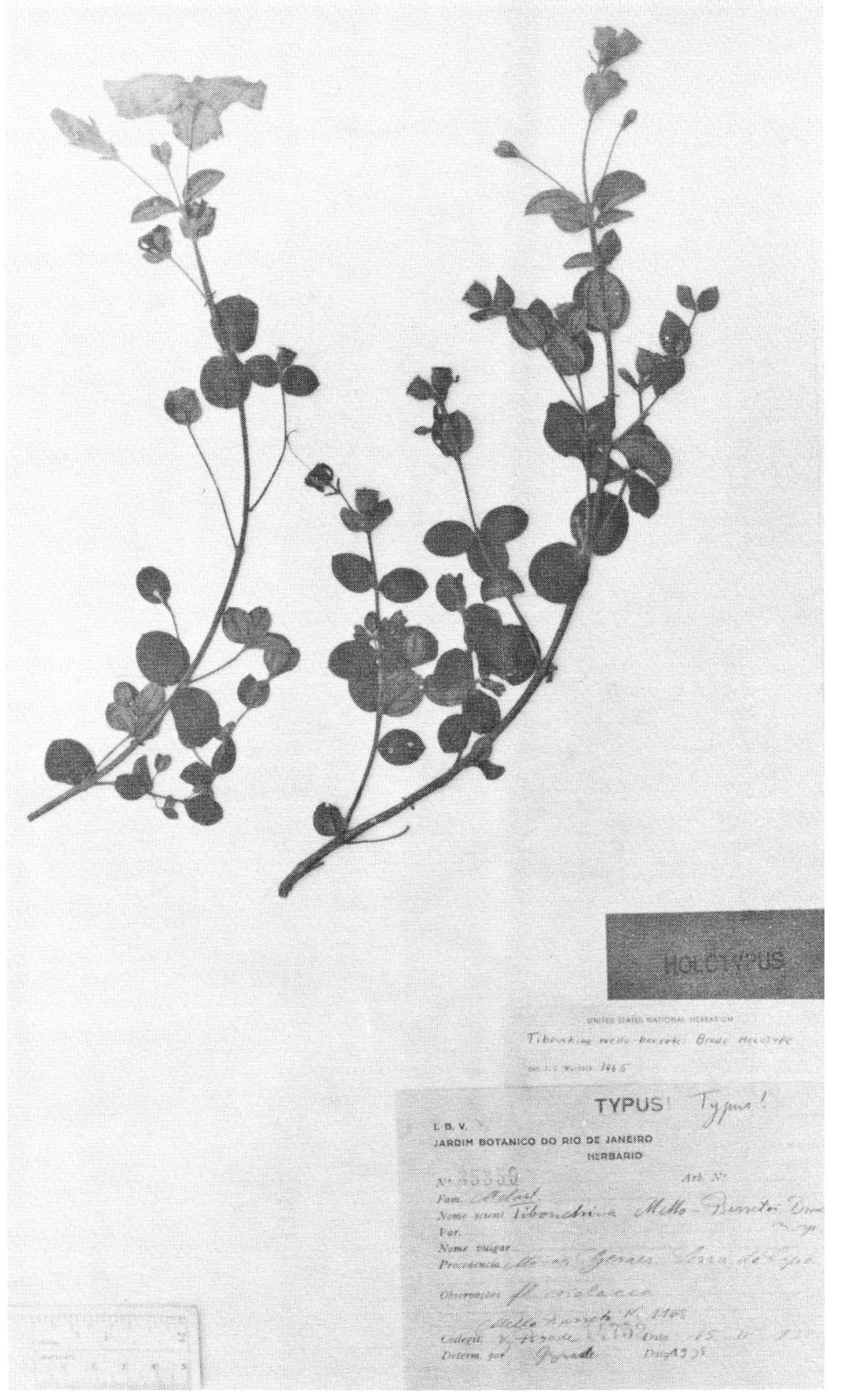

Foto 5 - Tibouchina Mello-Barretoi Brade 


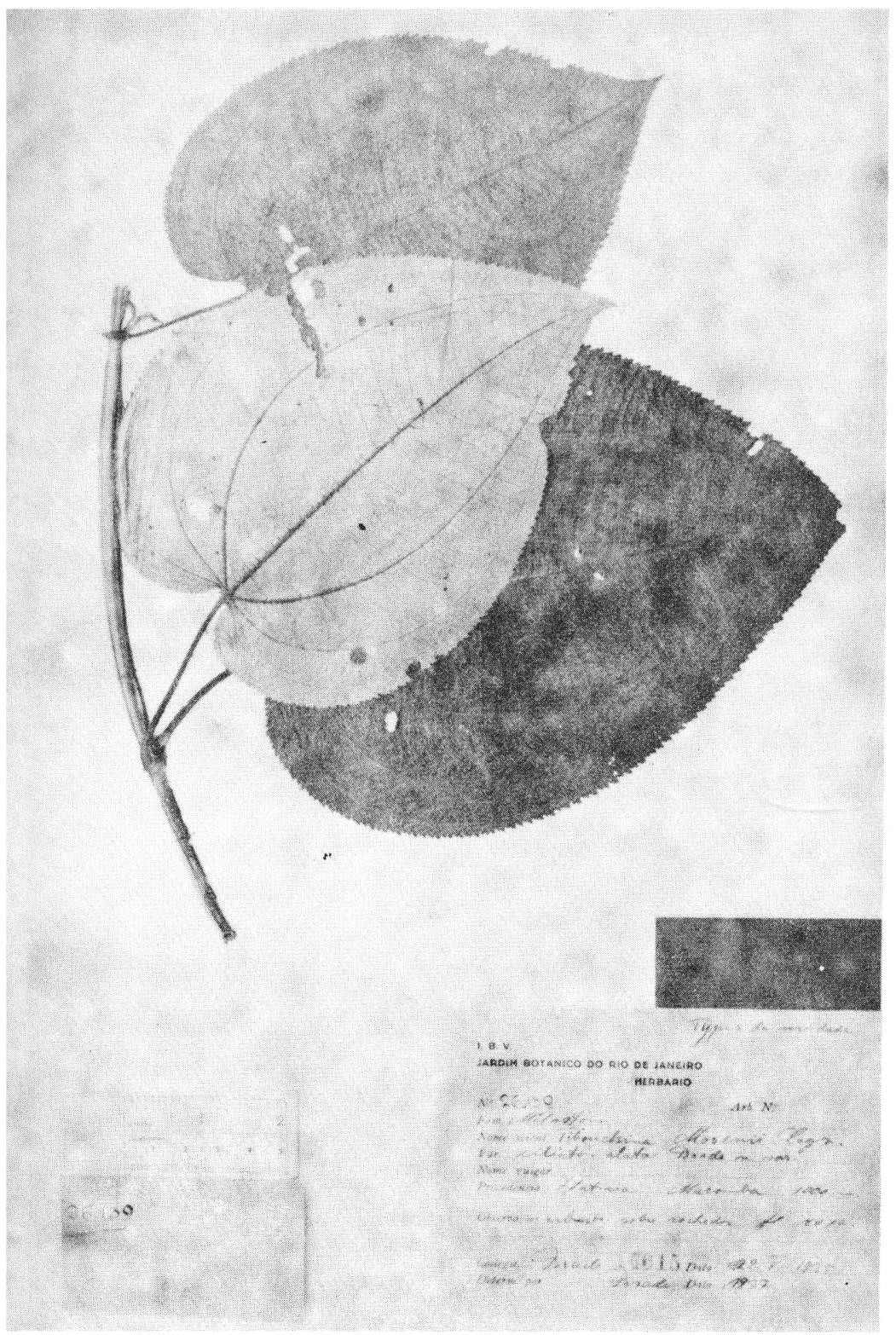

Foto 6 - Tibouchina Mosenii Cogniaux var. ciliato-alata Brade 


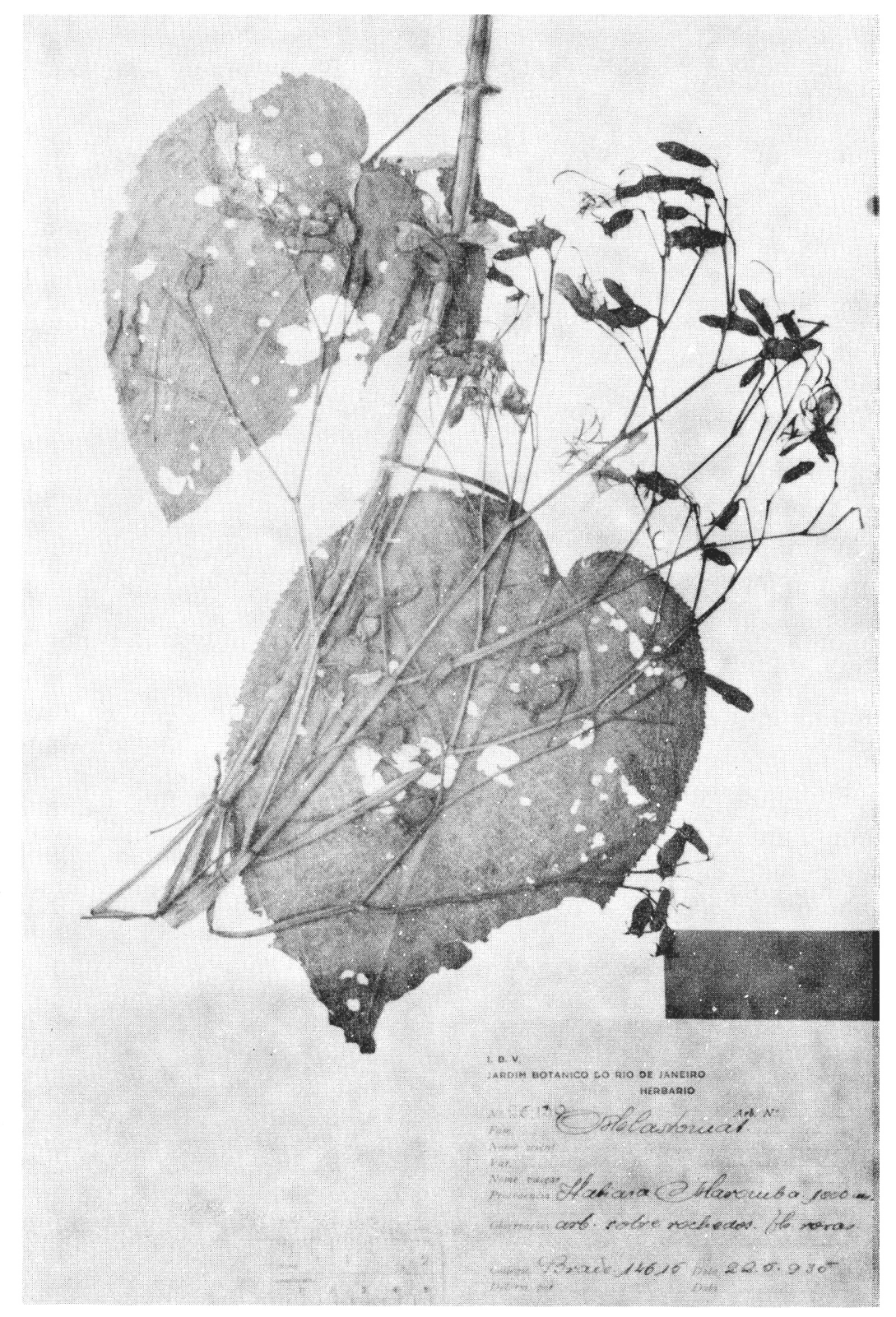

Foto 7 - Tibouchina Mosenii Cogniaux var. ciliato-alata Brade 


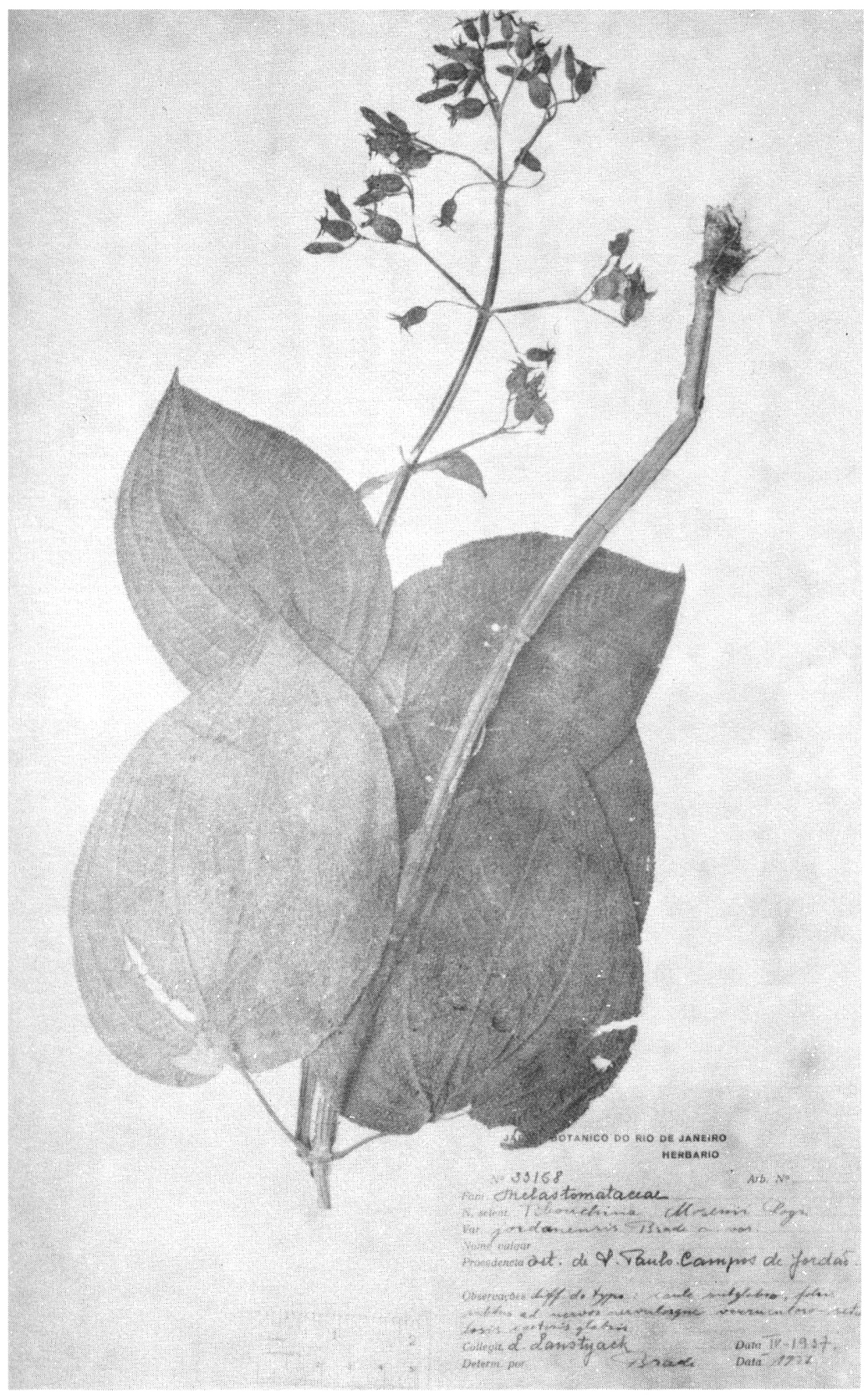

Foto 8 - Tibouchina Mosenii Cogniaux var. jordanensis Brade 


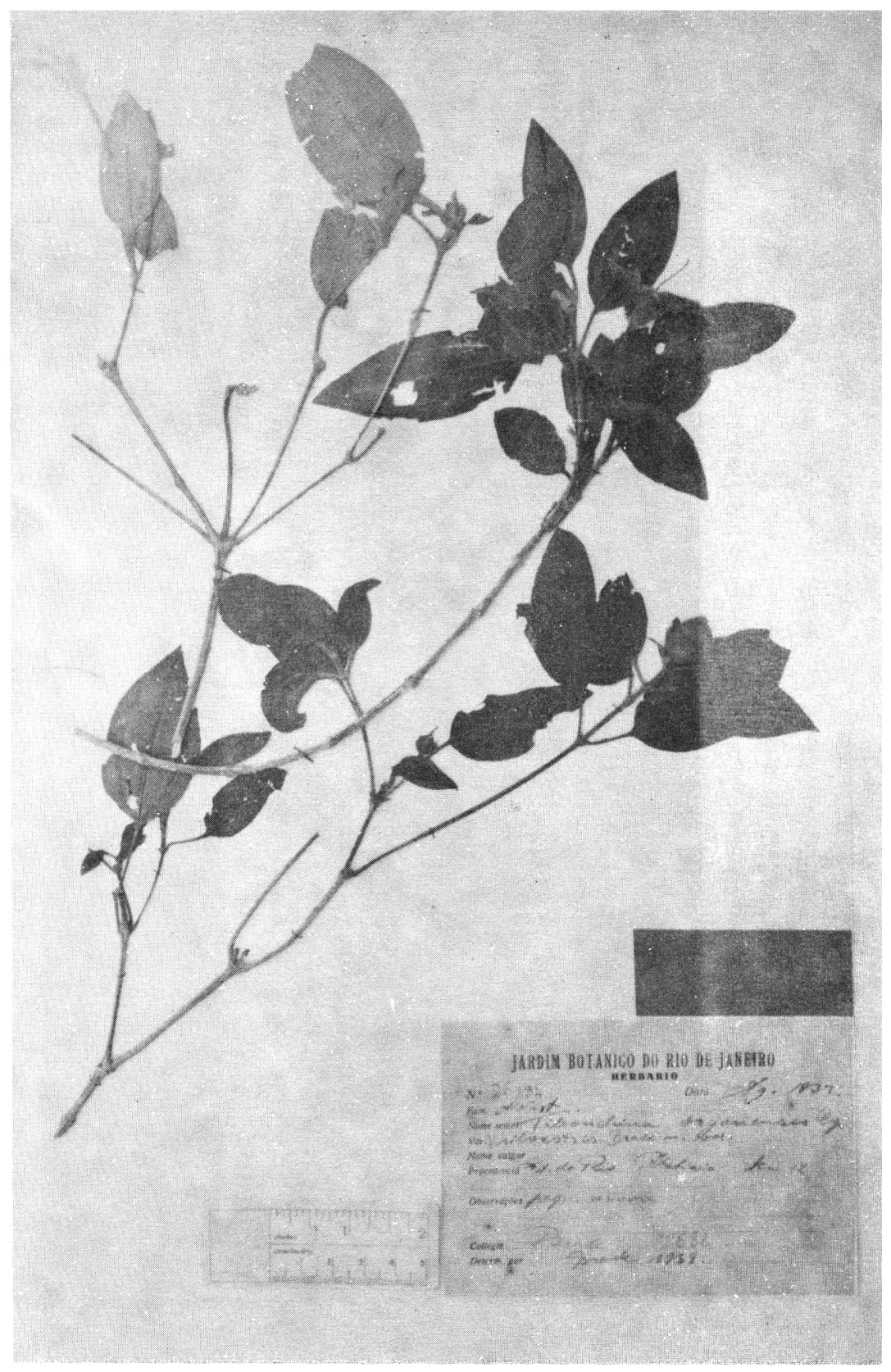

Foto 9 - Tibouchina organensis Cogniaux var. silvestris Brade 


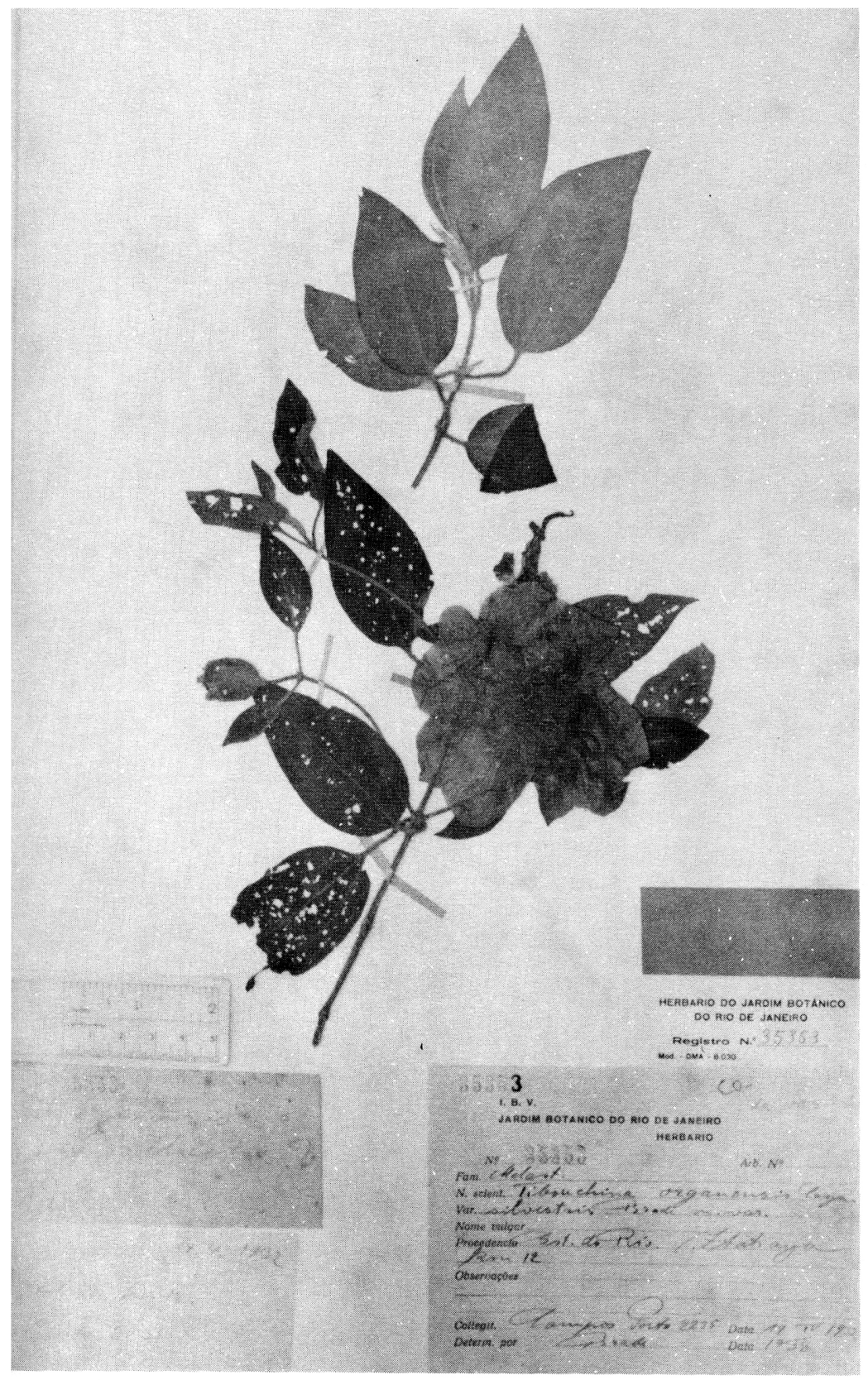

Foto 10 - Tibouchina organensis Cogniaux var. silvestris Brade 


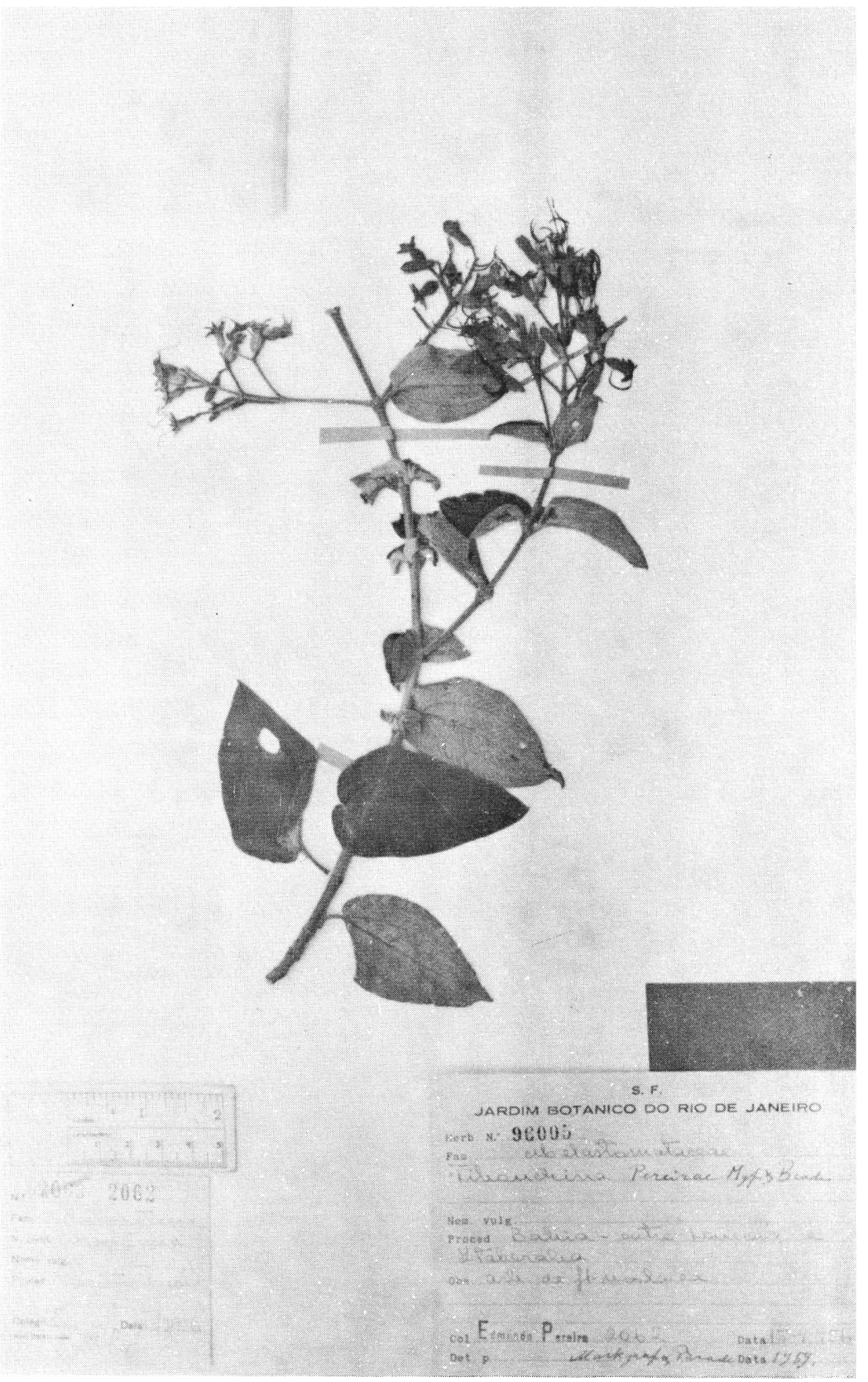

Foto 11 - Tibouchina Pereirae Brade 


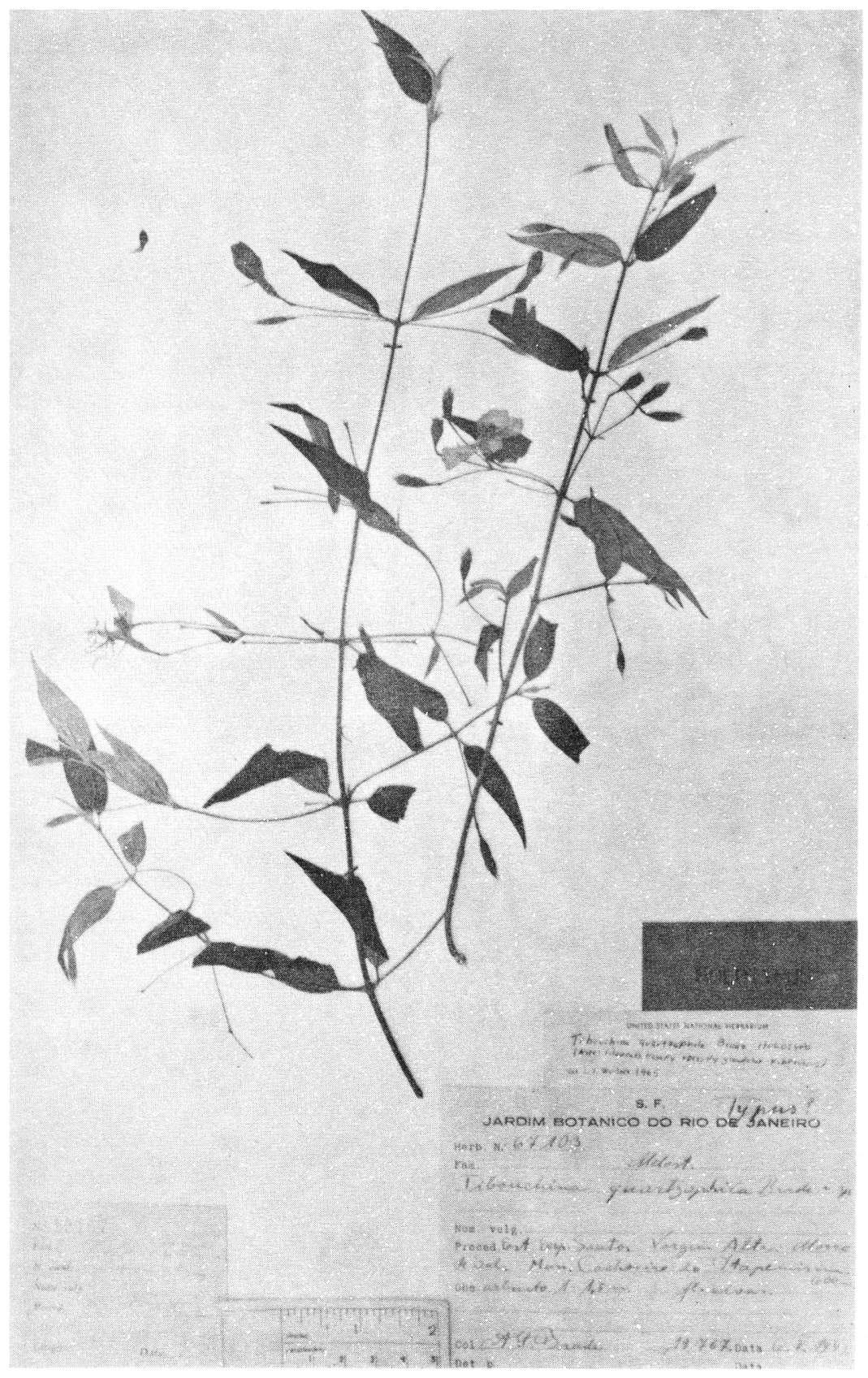

Foto 12 - Tibouchina quartzophila Brade 


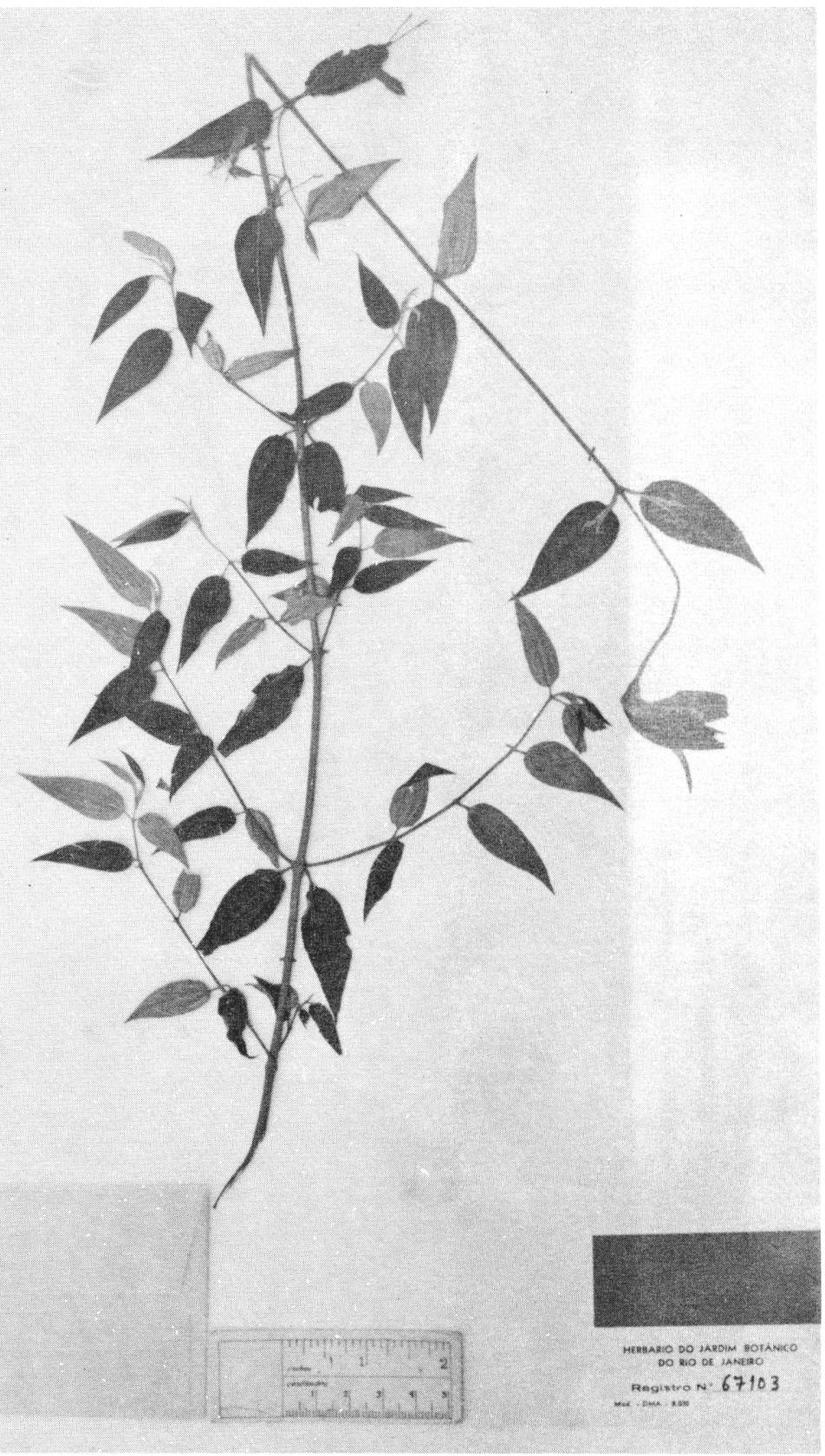

Foto 13 - Tibouchina quartzophila Brade 


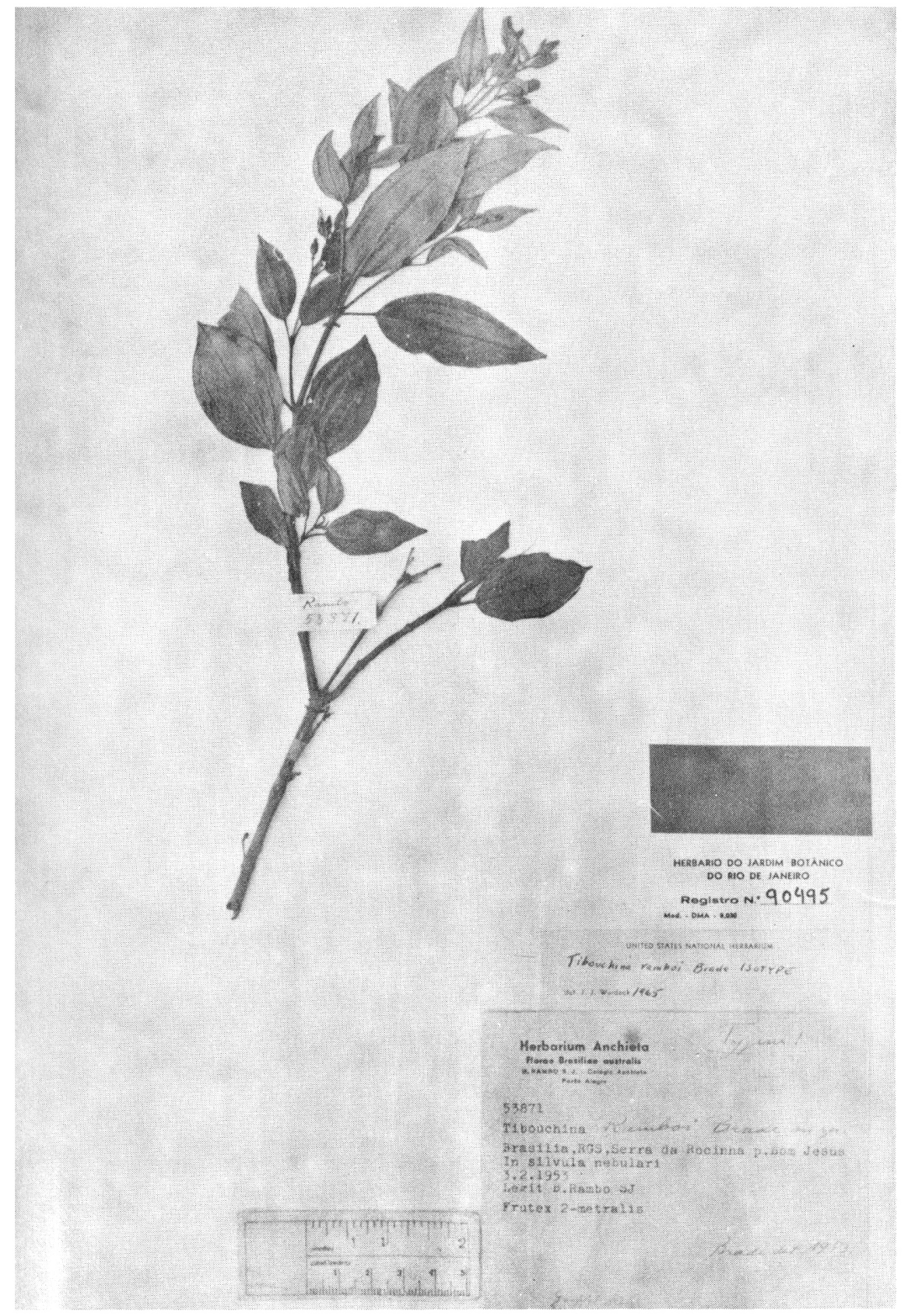

Foto 14 - Tibouchina ramboi Brade 


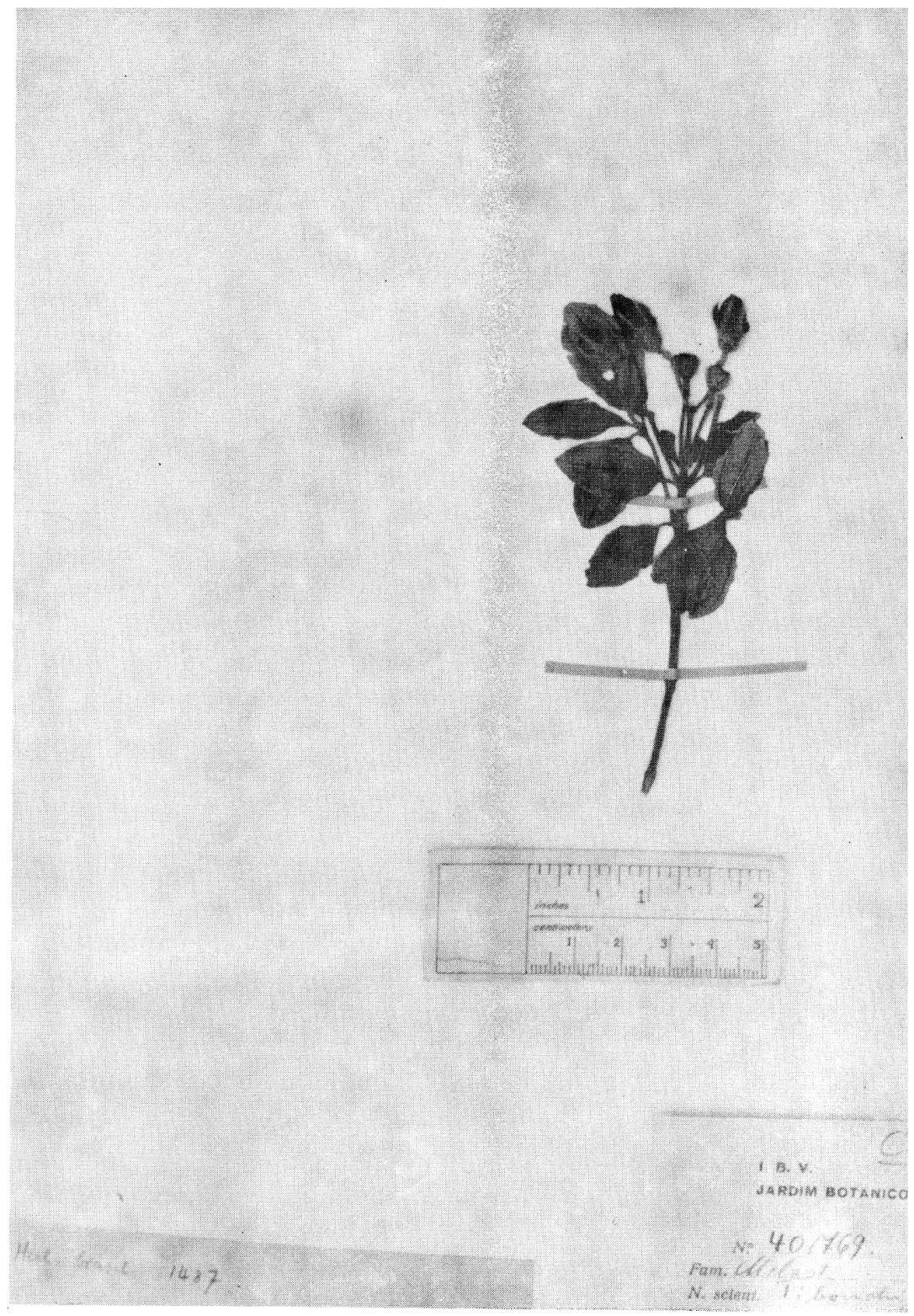

Foto 15 - Tibouchina Schenckii Cogniaux 


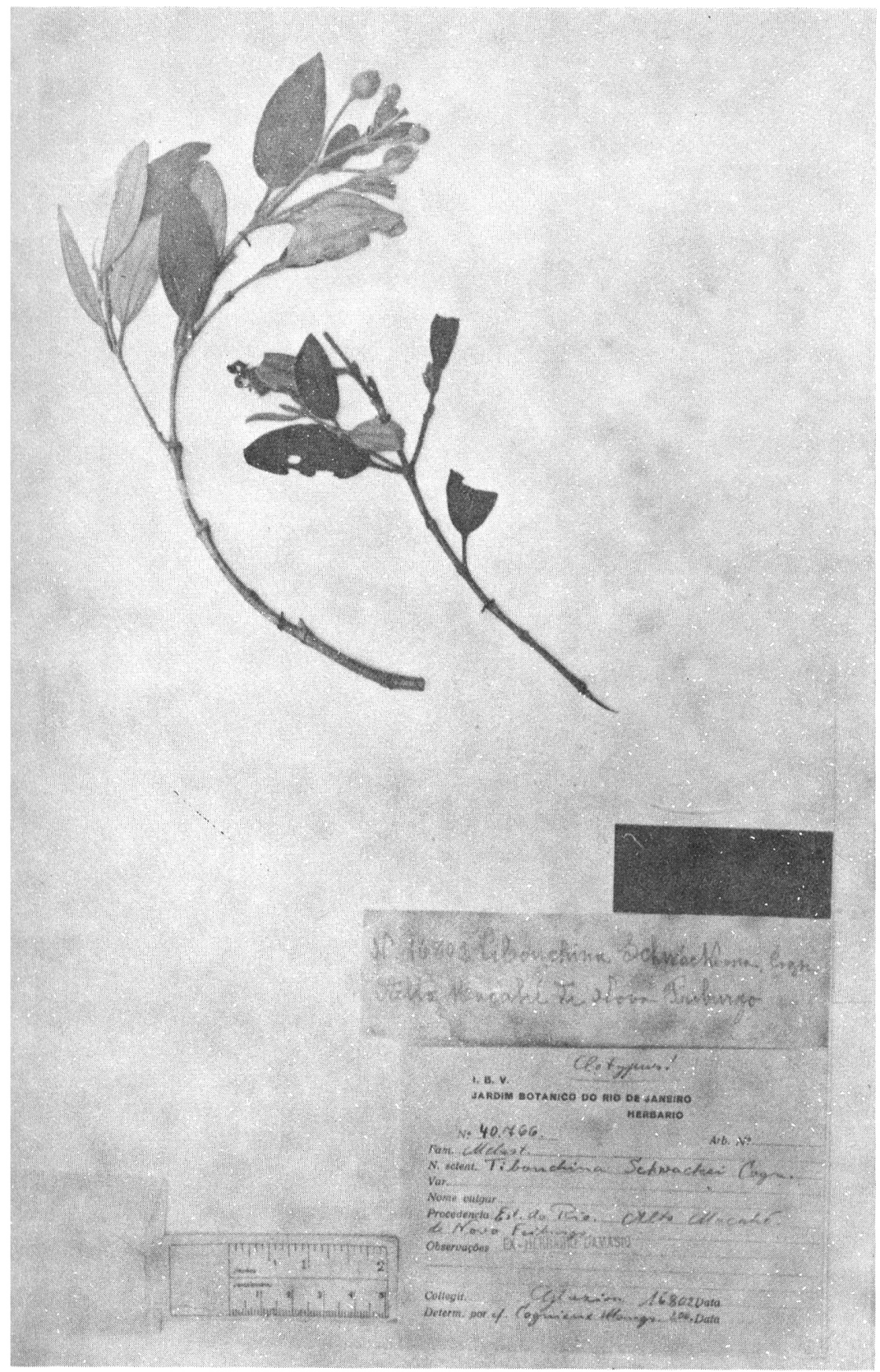

Foto 16 - Tibouchina Schwackei Cogniaux 


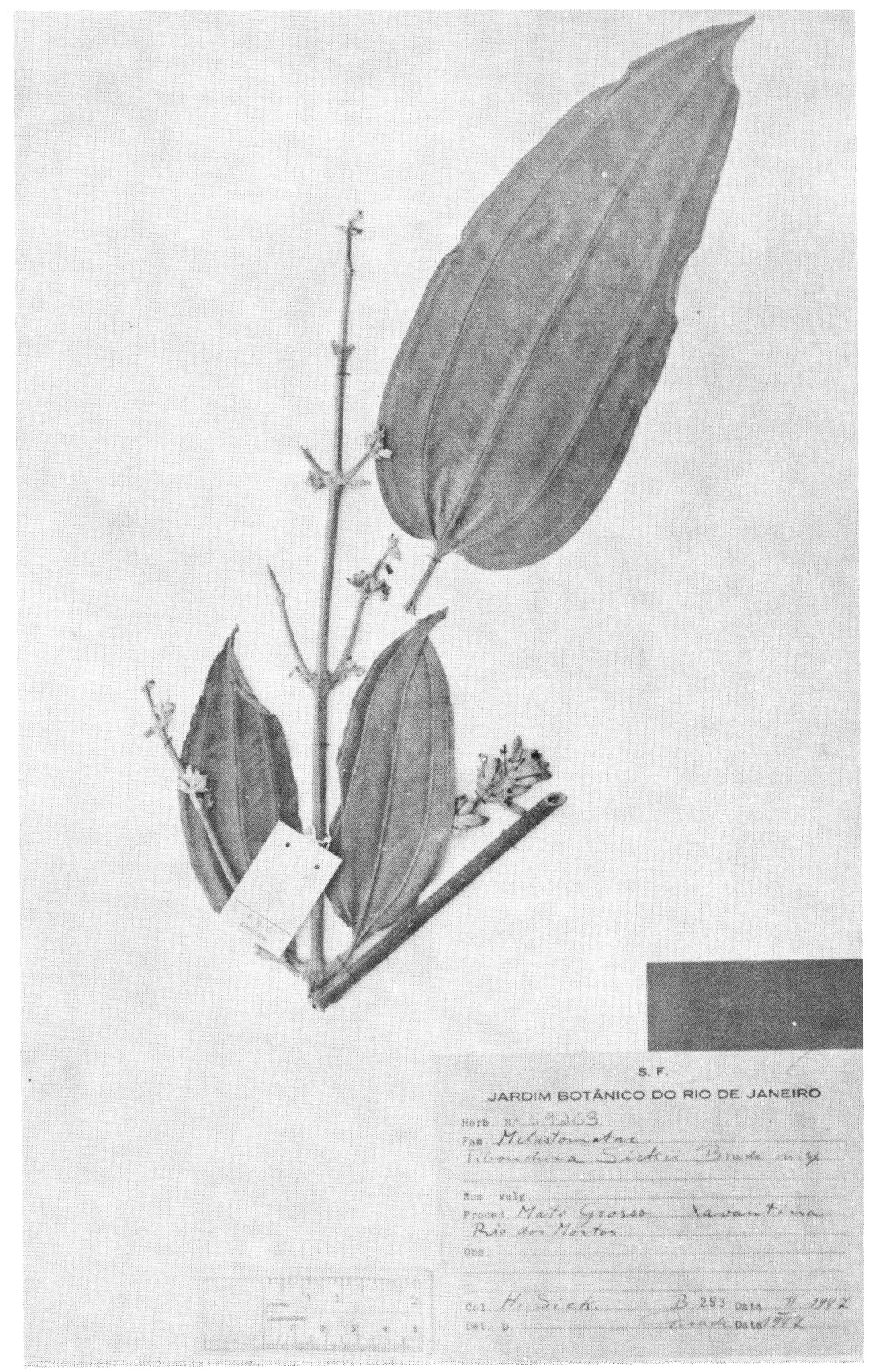

Foto 17 - Tibouchina sickii Brade 


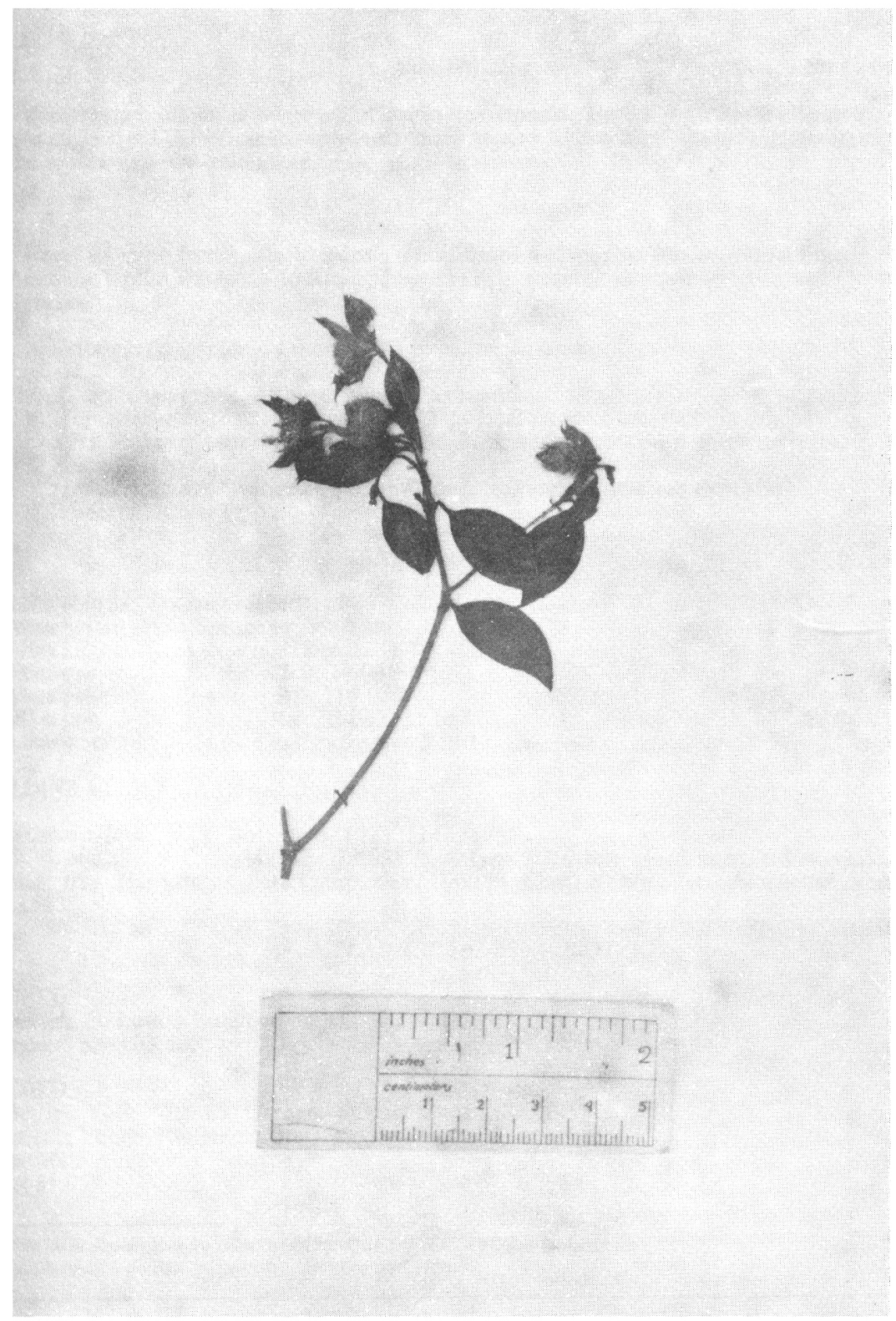

Foto 18 - Tibouchina Weddellii (Naudin) Cogniaux var. parvifolia Cogniaux 